Revista Destaques Acadêmicos, Lajeado, v. 10, n. 1, 2018. ISSN 2176-3070

DOI: http://dx.doi.org/10.22410/issn.2176-3070.v10i1a2018.1707

http://www.univates.br/revistas

\title{
GESTÃO ESTRATÉGICA EM EMPRESAS FAMILIARES: ANÁLISE DAS PESQUISAS QUALITATIVAS PUBLICADAS ENTRE 2010 E 2015
}

\author{
Denise Casagrande da Rocha ${ }^{1}$, Gabriel Machado Braido ${ }^{2}$, \\ Bernardete Bregolin Cerutti ${ }^{3}$
}

Resumo: Este artigo busca responder a seguinte questão: como são desenvolvidas as pesquisas qualitativas sobre gestão estratégica em empresas familiares? Para tanto, foram analisados 22 estudos publicados no período entre 2010 e 2015 obtidos a partir de uma busca nas bases de dados EBSCO Host e ISI Web of Knowledge. Como principais temas investigados por estes estudos, identificou-se: gestão estratégica, governança corporativa, gestão operacional, gestão de pessoas, cultura organizacional e gestão financeira. Quanto ao desenvolvimento das pesquisas, verificou-se que as estratégias utilizadas foram os estudos de caso, estudos teóricos e estudos narrativos e as principais técnicas de coleta de dados empregadas foram entrevistas, pesquisa documental, observações e grupos focais.

Palavras-chave: Gestão estratégica. Empresas familiares. Pesquisa qualitativa.

\section{INTRODUÇÃO}

A gestão estratégica constitui-se em um campo acadêmico relativamente recente, que possui fragilidades no consenso sobre os próprios limites e significados, bem como concorre e apresenta sobreposições com outros campos mais fortes como a economia, a sociologia, o marketing, as finanças e a psicologia (NAG; HAMBRICK; CHEN, 2007). Porém, parece não haver dúvidas quanto à

1 Doutoranda em Administração (PPGAdm/Unisinos) e Mestre em Administração (PPGA/ EA/UFRGS). É diretora Executiva da Denise Casagrande - Desenvolvimento de Pessoas e Organizações.denise@denisecasagrande.com.br

2 Doutorando em Administração (PPGAdm/Unisinos) e Mestre em Administração (PPGA/ EA/UFRGS). É professor assistente da Universidade do Vale do Taquari - Univates. gabrielb@univates.br

3 Doutoranda em Desenvolvimento Regional (PPGDR/Unisc) e Mestre em Ambiente e Desenvolvimento (PPGAD/Univates). E professora assistente da Universidade do Vale do Taquari - Univates. bcerutti@univates.br 
relevância e contribuição da gestão estratégica, tanto para fins do alinhamento dos esforços em direção ao conjunto de objetivos, como para a formulação, implementação e controle do progresso destes, em empresas familiares ou não familiares (SHARMA; CHRISMAN; CHUA, 1997).

No que se refere ao reconhecimento da área de empresas familiares como um campo acadêmico, a situação mostra-se semelhante. Curiosamente, embora a histórica existência de empresas constituídas por famílias, consta que este tema passou a ser tratado com algum formalismo acadêmico somente após os anos 60, por iniciativa de estudiosos interessados em compreender melhor as particularidades deste tipo de arranjo produtivo, o que foi fortalecido posteriormente pela criação do Family Firm Institute (FFI), em 1986, e a Family Business Revieww, em 1988 (SHARMA; CHRISMAN; GERSICK, 2012).

Embora desde então já tenham ocorrido importantes avanços nesta trajetória, percebe-se que os temas correlatos a empresas familiares ainda carecem de reconhecimento como integrantes de uma disciplina independente, suficientemente robusta para caracterizar um campo acadêmico (STEWART; MINER, 2011). Em verdade, ao utilizar-se de teorias de outras disciplinas acadêmicas para explicar os fenômenos típicos das empresas familiares, os pesquisadores acabam não se apropriando suficientemente das questões pertinentes a este campo de pesquisa, oferecem contribuições acanhadas para o desenvolvimento de teorias próprias e como consequência reafirmam o relativo atraso desta construção, tendo em vista as demandas do contexto de negócios (BIRD et al., 2002). Neste sentido, aproximar as perspectivas de gestão estratégica e de empresas familiares representa uma oportunidade real de compartilhamento de ideias, discussão teórica e aplicada, reforçando, sobretudo, o amadurecimento destas respectivas áreas de pesquisa.

Durante a década de 1990, o campo de produção científica em Administração parece ter se voltado para si mesmo, em uma análise crítica que objetivou apurar a qualidade, o rigor, a relevância e a originalidade dos diversos artigos publicados na área (CALDAS; TONELLI; LACOMBE, 2002). Devido ao aumento significativo na quantidade de publicações observado nas últimas décadas, torna-se cada vez mais necessária a condução de balanços críticos e avaliações da qualidade em relação à quantidade dos trabalhos produzidos, bem como se estes avançam, de fato, no conhecimento considerando as lacunas e as oportunidades apontadas por estudos anteriores (SOUZA; REINERT; SPROSSER, 2009).

Diante disso, surgiram os estudos bibliográficos, que se multiplicaram principalmente nos setores administrativos, marketing, sistemas de informação, administração pública e produção, analisando as mais diversas dimensões da produção científica em cada uma dessas áreas (CALDAS; TONELLI; LACOMBE, 2002). Souza, Reinert e Sprosser (2009) constataram que a partir da comparação desses estudos nas diversas áreas da Administração, a mais frequente é a de "estratégia em organizações", especialmente pelas temáticas relacionadas com 
estratégia, competitividade e vantagem competitiva. Contudo, embora existam importantes publicações acerca destes temas, é necessário, sistematicamente, revisitar a literatura existe, bem como contribuir para a consolidação das pesquisas sobre a gestão estratégica em empresas familiares, o que será foco do presente estudo.

Para fins da concepção e realização de uma pesquisa em todas as suas etapas, a escolha do método a ser utilizado é um tema central para a maioria dos pesquisadores. O método de pesquisa é o "fio condutor" que permite ao pesquisador conectar os objetivos iniciais do estudo às conclusões com o rigor científico e com a ética necessárias ao avanço do conhecimento. Com características distintas (estratégias de investigação, foco e unidade de análise, amostragem, coleta de dados, entre outras) e para responder a diferentes objetivos e problemas de pesquisa, o método de pesquisa pode ser quantitativo, qualitativo e misto (englobando os métodos qualitativo e quantitativo). A pesquisa qualitativa, foco deste estudo, permite ao pesquisador obter uma melhor visão e compreensão do problema, na medida em que ela busca gerar hipóteses e identificar variáveis que devem ser incluídas no estudo, explorando-o com poucas ideias acerca do resultado dessa investigação (MALHOTRA, 2012). Embora busquem o que é comum, as pesquisas qualitativas mantêm-se abertas para entender os significados e a singularidade que cada indivíduo atribui à realidade ( $\mathrm{ROESCH}, 2009)$.

Diante do contexto apresentado surge a seguinte questão de pesquisa: Como são desenvolvidas as pesquisas qualitativas sobre gestão estratégica em empresas familiares? Para responder a esta questão, este artigo tem como objetivos: (i) verificar o que foi publicado sobre gestão estratégica em empresas familiares no período entre 2010 e 2015; (ii) analisar, entre os estudos identificados, aqueles que adotaram uma abordagem metodológica qualitativa; (iii) analisar as estratégias de pesquisa e as técnicas de coleta de dados utilizadas no desenvolvimento das pesquisas qualitativas; e (iv) identificar as limitações metodológicas apontadas pelos autores nos artigos analisados.

Espera-se que este artigo seja uma contribuição para os pesquisadores interessados na temática de gestão estratégica em empresas familiares, apresentando um panorama das pesquisas qualitativas já realizadas, bem como algumas limitações do método qualitativo de investigação.

\section{PESQUISA QUALITATIVA}

No que se refere à pesquisa qualitativa, é importante destacar que por tratar-se de um método marcadamente contextual e voltado para apuração realista dos fenômenos em um período de tempo (por vezes longo), permite ao pesquisador obter melhor visão e compreensão do problema, na medida em que busca gerar hipóteses e identificar variáveis que devem ser incluídas no estudo, explorando-o de maneira aberta, com poucas ideias prévias acerca 
dos resultados da investigação (MALHOTRA, 2012). Embora busquem o que é comum, as pesquisas qualitativas mantêm-se abertas para entender os significados e a singularidade que cada indivíduo atribui à realidade $(\mathrm{ROESCH}$, 2009).

De acordo com Malhotra (2012), os pesquisadores utilizam o método qualitativo para definir o problema ou desenvolver uma abordagem, gerando hipóteses e identificando variáveis que devem ser incluídas na pesquisa. O autor ainda acrescenta que a pesquisa qualitativa visa uma melhor visão e compreensão do contexto do problema, sendo que para o embasamento desse tipo de pesquisa são utilizadas amostras pequenas e os seus dados não são analisados de forma estatística (MALHOTRA, 2005).

Creswell (2010) e Gray (2012) apontam como características gerais do método qualitativo: o campo de pesquisa é real, onde ocorre o problema ou questão que se deseja entender; forte contato do pesquisador com o campo de pesquisa; utilização de múltiplas fontes de dados; análise indutiva dos dados coletados; os participantes conferem significados ao problema ou questão de pesquisa; utilização de teorias para embasar a pesquisa; forma de investigação interpretativa dos achados da pesquisa; visão panorâmica e holística do campo pesquisado; possibilidade de fazer a verificação (revisão) de temas emergentes com os próprios pesquisados.

Neste sentido, a pesquisa qualitativa é permanentemente exploratória, de tal maneira que as dúvidas, as respostas, as pistas e os novos territórios de indagação permanecem abertos até o final (CASTRO,2006). O método qualitativo pressupõe a utilização (individual ou combinada) das seguintes estratégias de investigação: estudos de caso, etnografia, etnometodologia, fenomenologia, teoria fundamentada, pesquisa ação participante, análise narrativa, análise bibliográfica, estudos culturais e estudos de gênero (CRESWELL, 2010).

A coleta de dados neste tipo de pesquisa pode ocorrer através da observação participativa do pesquisador do objeto de estudo, da realização de entrevistas (face-a-face, telefone, e-mail ou grupos focais), da análise de documentos (públicos ou privados) ou ainda da análise de outros materiais audiovisuais (fotografias, filmes, softwares, entre outros). A utilização combinada destas alternativas para a coleta de dados, visa a conferir maior sentido e confiança à pesquisa (CASTRO, 2006; GRAY, 2012).

Não são raros os debates entre estudiosos, envolvendo questões relacionadas a confiabilidade, validade e generalizabilidade da pesquisa qualitativa. O envolvimento pessoal do pesquisador é tão intenso na interpretação dos dados coletados que, por vezes, uma mesma base de dados coletados, analisada por diferentes pesquisadores pode gerar conclusões inteiramente distintas (MAYS, 1995).

Neste sentido a subjetividade intrínseca ao método e a dificuldade em ampliar o entendimento do contexto analisado oportunizando a reprodução e 
a aplicação das conclusões, são algumas das críticas mais frequentes a pesquisa qualitativa. Em contrapartida, os pesquisadores qualitativos, preocupam-se em reafirmar e demonstrar o rigor científico do método, aprimorando estratégias e ferramentas de investigação que garantam a cientificidade deste (GRAY, 2012).

\section{PROCEDIMENTOS METODOLÓGICOS}

Para a identificação das pesquisas qualitativas que foram analisadas na temática de gestão estratégia em empresas familiares, seguiu-se as etapas sugeridas por Dresch, Lacerda e Antunes (2015), a saber: (1) Definição do tema ou questão de pesquisa; (2) Busca dos estudos existentes; (3) Seleção dos estudos para integrar a pesquisa; (4) Avaliação da relevância e da qualidade dos estudos selecionados; e (5) Discussão dos resultados obtidos.

\section{Etapa 1 - Definição do tema ou questão de pesquisa}

Para a realização da pesquisa, definiu-se como problema a seguinte questão: como são desenvolvidas as pesquisas qualitativas sobre gestão estratégica em empresas familiares? Neste sentido, por meio da realização deste estudo, pretende-se aprofundar os conhecimentos sobre a temática central, bem como recolher evidências sobre a evolução das pesquisas qualitativas (abordagens teóricas, estratégias de pesquisa, técnicas de coletas de dados, principais temas investigados e limitações metodológicas) sobre gestão estratégica em empresas familiares.

\section{Etapa 2 - Busca dos estudos existentes}

Nesta etapa definiu-se, primeiramente, um roteiro com os critérios a serem adotados para a busca dos estudos disponíveis sobre o tema definido. Para a seleção de artigos, utilizou-se a busca avançada por tópico, com as seguintes palavras-chave: "strategic management" AND "family firms", compreendendo artigos publicados em periódicos ou jornais acadêmicos, em Língua Inglesa ou Espanhola no período entre 2010 e 2015, nas bases de dados EBSCO Host e ISI Web of Knowledge. O mapeamento ocorreu em 23 de outubro de 2015, onde identificou-se um total de 107 publicações, sendo 65 provenientes da EBSCO Host e 42 da ISI Web of Knowledge.

\section{Etapa 3 - Seleção dos estudos para integrar a pesquisa}

Com o objetivo de refinar a amostra de publicações, nesta etapa foram analisados todos os resumos e palavras-chave dos artigos selecionados, a fim de identificar aqueles de fato relacionados à temática de gestão estratégia em empresas familiares, procedendo também a exclusão da amostra de publicações relativas a casos de ensino. 
Buscando diminuir a subjetividade e os vieses dos pesquisadores na seleção dos artigos pela análise do resumo, esta foi realizada individualmente pelos autores e, na sequência, os resultados foram confrontados. Os casos de discrepância foram individualmente analisados, buscando o consenso sobre a manutenção ou exclusão do artigo. Após esta análise, foram selecionadas 39 publicações. De posse desta amostra, passou-se para a análise da abordagem metodológica de cada artigo, classificando-os em estudos qualitativos, quantitativos ou mistos. Foram identificados 21 estudos com abordagem qualitativa, 17 estudos quantitativos e 1 estudo com abordagem mista. Visando ao alcance dos objetivos propostos nesta pesquisa, foram selecionados para análise os 21 artigos classificados como qualitativos e o artigo classificado como misto, resultando em uma amostra final de 22 artigos. A autoria e o ano de publicação de cada artigo analisado são apresentados no Quadro 1, sendo que as referências completas podem ser encontradas na seção de referências ao final deste documento.

Quadro 1 - Artigos selecionados para este estudo

\begin{tabular}{|c|l|c|l|}
\hline $\mathbf{N}^{\mathbf{0}}$ & \multicolumn{1}{|c|}{ Autoria (ano) } & $\mathbf{N}^{\mathbf{0}}$ & \multicolumn{1}{|c|}{ Autoria (ano) } \\
\hline $\mathbf{1}$ & LOZANO (2015) & $\mathbf{1 2}$ & SHARMA; CHUA (2013) \\
\hline $\mathbf{2}$ & $\begin{array}{l}\text { DE CLERCQ; BELAUSTEGUIGOITIA } \\
\text { (2015) }\end{array}$ & $\mathbf{1 3}$ & YU et al. (2013) \\
\hline $\mathbf{3}$ & ROMERO et al. (2014) & $\mathbf{1 4}$ & JONES et al. (2013) \\
\hline $\mathbf{4}$ & WRIGHT et al. (2014) & $\mathbf{1 5}$ & HOLT (2012) \\
\hline $\mathbf{5}$ & DASPIT; LONG (2014) & $\mathbf{1 6}$ & BOYD; HOLLENSEN (2012) \\
\hline $\mathbf{6}$ & KOTLAR; MASSIS (2013) & $\mathbf{1 7}$ & AGUILAR; LEMA (2011) \\
\hline $\mathbf{7}$ & MEMILI; WELSH; LUTHANS (2013) & $\mathbf{1 8}$ & SHARMA; SALVATO (2011) \\
\hline $\mathbf{8}$ & DeTIENNE; CHIRICO (2013) & $\mathbf{1 9}$ & ALTINDAG; ZEHIR; ACAR (2011) \\
\hline $\mathbf{9}$ & CHRISMAN et al. (2013) & $\mathbf{2 0}$ & LUMPKIN; BRIGHAM (2011) \\
\hline $\mathbf{1 0}$ & JASKIEWICZ; LUCHAK (2013) & $\mathbf{2 1}$ & LUMPKIN; STEIER; WRIGHT (2011) \\
\hline $\mathbf{1 1}$ & LIONZO; ROSSIGNOLI (2013) & $\mathbf{2 2}$ & O'REGAN et al. (2010) \\
\hline
\end{tabular}

Fonte: elaborado pelos autores.

\section{Etapa 4 - Avaliação da relevância e da qualidade dos estudos selecionados}

Para a avaliação da relevância dos estudos selecionados, adotouse a verificação do Fator de Impacto, no Journal Citation Reports, do Web of Knowledge, de cada periódico onde os artigos foram publicados, bem como a leitura completa do artigo para verificar o nível de enquadramento do mesmo ao escopo desejado para pesquisa.

Ressalta-se que alguns artigos selecionados estão publicados em revistas sem fator de impacto, porém conforme orientação de um especialista da área de gestão estratégica em empresas familiares, optou-se por mantê-los no estudo, 
devido a muitas revistas serem novas e ainda não terem fator de impacto definido, fato que não deve influenciar na qualidade dos estudos publicados.

\section{Etapa 5 - Discussão dos resultados obtidos}

Para verificação dos artigos e discussão dos resultados, recolheuse evidências nos artigos selecionados no que se refere à autoria, periódico de publicação, fator de impacto, número de citações, abordagens teóricas, estratégias de investigação, técnicas de coleta de dados utilizadas, bem como as limitações metodológicas apresentadas nos estudos.

\section{APRESENTAÇÃO E DISCUSSÃO DOS RESULTADOS}

A partir da análise dos temas investigados nos artigos analisados, identificou-se cinco blocos temáticos nos estudos qualitativos sobre gestão estratégica em empresas familiares, conforme a frequência nos artigos analisados, a saber: gestão estratégica, governança corporativa, gestão operacional, gestão de pessoas e cultura organizacional e gestão financeira.

De forma específica, cada bloco temático, engloba temas variados, mas correlatos ao tema central. No que se refere à gestão estratégica, as pesquisas exaltam temas como planejamento estratégico e gestão estratégica dos negócios; aprendizagem organizacional e gestão do conhecimento; competitividade e vantagem competitiva sustentável; inovação; além de capacidades dinâmicas; crescimento, internacionalização e globalização da empresa; estratégias de marketing; aquisições; competição e cooperação, entre outros. Já nos estudos sobre governança corporativa são temas centrais orientação empreendedora, empreendedorismo e empreendimentos familiares; transição de gerações e planejamento de sucessão; governança corporativa e participativa; estrutura societária e propriedade da empresa e da família; gestão, controle e compromisso familiar; heterogeneidade e ciclo de vida das empresas familiares, entre outros.

$\mathrm{O}$ foco dos estudos relacionados à gestão de pessoas e cultura organizacional, refere-se a temas como os valores da família e da empresa; redes, laços e interações sociais entre a família e os profissionais; capital humano, social e emocional da empresa familiar; competências e eficiência; conflitos familiares; compromisso coletivo; confiança e comportamento organizacional. Já nos estudos sobre gestão operacional evidencia-se uma visão mais de curto prazo, orientada para metas, indicadores e desempenho da empresa; gestão de recursos; análise comparativa do desempenho dos CEOs familiares versus não-familiares; gestão industrial, produtividade e eficiência operacional. Por fim, os artigos sobre gestão financeira compreendem principalmente temáticas relacionadas à análise de atividades/oportunidades econômicas; investimentos gerais e investimentos específicos em pesquisa e desenvolvimento; segurança e risco econômico financeiro da empresa; endividamento, gestão de caixa e geração de valor econômico. 


\subsection{Abordagens teóricas}

Nesta etapa da pesquisa buscou-se identificar as abordagens teóricas que sustentaram os estudos analisados. Diante disso, verificou-se que 9 artigos $(40,90 \%$ do total) não utilizaram abordagens teóricas para embasamento dos estudos, enquanto que os outros 13 artigos (59,10\% do total) utilizaram 12 abordagens teóricas diferentes, as quais são apresentadas na Tabela 1, juntamente com a sua frequência de utilização. Vale ressaltar que alguns pesquisadores utilizam uma combinação de abordagens teóricas em suas análises, fato que justifica a frequência total de 19 abordagens teóricas presentes em 13 dos artigos analisados.

Tabela 1 - Abordagens teóricas utilizadas nos artigos analisados

\begin{tabular}{l|c|c|c}
\hline \multicolumn{1}{c|}{ Abordagem teórica } & Qtde. & $\mathbf{\%}$ & $\mathbf{\%}$ acum. \\
\hline Visão Baseada em Recursos (RBV) & 4 & $21,05 \%$ & $21,05 \%$ \\
\hline Capacidades Dinâmicas & 2 & $10,53 \%$ & $31,58 \%$ \\
\hline Teoria do Comportamento da Firma & 2 & $10,53 \%$ & $42,11 \%$ \\
\hline Teoria Institucional & 2 & $10,53 \%$ & $52,63 \%$ \\
\hline Teoria Prospectiva & 2 & $10,53 \%$ & $63,16 \%$ \\
\hline Teoria da Imagem & 1 & $5,26 \%$ & $68,42 \%$ \\
\hline Teoria da Aprendizagem Organizacional & 1 & $5,26 \%$ & $73,68 \%$ \\
\hline Teoria da Contingência & 1 & $5,26 \%$ & $78,95 \%$ \\
\hline Teoria da Escolha Estratégica & 1 & $5,26 \%$ & $84,21 \%$ \\
\hline Teoria da Regulamentação & 1 & $5,26 \%$ & $89,47 \%$ \\
\hline Teoria da Troca Social & 1 & $5,26 \%$ & $94,74 \%$ \\
\hline Teoria dos Stakeholders & 1 & $5,26 \%$ & $100,00 \%$ \\
\hline
\end{tabular}

Fonte: elaborada pelos autores.

A Visão Baseada em Recursos (RBV) destaca-se como a abordagem mais recorrente, tendo sido utilizada em 4 estudos (21,05\% do total). Conforme Barney e Hesterly (2007), na perspectiva da RBV, as estratégias são definidas com vistas à potencialização dos ativos internos (recursos e capacidades controlados pela empresa). A teoria das Capacidades Dinâmicas foi utilizada em 2 estudos analisados, o correspondente a 10,53\% do total. As capacidades dinâmicas podem ser consideradas como as ferramentas utilizadas para manipular as configurações existentes nas organizações a fim de criar novos recursos (EISENHARDT; MARTIN, 2000).

A Teoria Institucional preocupa-se com a forma como as organizações protegem e melhoram as suas posições e a sua legitimidade, em conformidade com as regras e normas estabelecidas no ambiente institucional (MEYER; 
ROWAN, 1991). Neste contexto, o termo "instituição" refere-se aos conjuntos formais de regras e acordos que as organizações e o indivíduos devem seguir (BRUTON; AHLSTRON; LI, 2010), as quais são derivadas de regras como as estruturas reguladoras, órgãos governamentais, leis, profissões e outras práticas sociais e culturais que exercem pressões de conformidade (DiMAGGIO; POWELL, 1983). Esta abordagem teórica foi utilizada em 10,53\% dos estudos qualitativos sobre gestão estratégica em empresas familiares.

Com base na Teoria Comportamental da Firma, vários pesquisadores de empresas familiares têm argumentado que as empresas familiares estão em uma posição única em relação às empresas não familiares no que se refere à busca de objetivos que reflitam os valores e aspirações do sistema familiar, tais como objetivos de riqueza socioemocionais (CHRISMAN et al., 2010; GOMEZMEJÍA et al., 2007). Neste estudo, observou-se que 2 artigos (10,53\% do total) foram embasados pela teoria comportamental da firma.

Outros 2 estudos foram conduzidos à luz da Teoria Prospectiva, a qual propõe que os tomadores de decisão comparam os resultados potenciais com um ponto de referência, e que o enquadramento desses resultados influencia na utilidade esperada (KAHNEMAN; TVERSKY, 1979). Berns, Laibson e Loewenstein (2007) afirmam que, de acordo com esta teoria, diante de uma decisão sobre aceitar um resultado atrasado, a escolha será feita de forma diferente se o resultado for cognitivamente interpretado como gratificante e agradável em comparação com algo frustrante ou fútil, ou seja, as representações evocam heurísticas de escolha específicas, que aumentam ou diminuem a importante das recompensas atrasadas.

Outras teorias, como Teoria da Imagem, da Aprendizagem Organizacional, da Contingência, da Escolha Estratégica, da Regulamentação, da Troca Social e dos Stakeholders foram utilizadas em 1 estudo cada $(5,26 \%)$. Diante dessa análise, entende-se que a variedade de teorias utilizadas nos artigos pode sugerir o interesse dos pesquisadores em abordar a perspectiva das empresas familiares a partir de diversas lentes teóricas, mas ao mesmo tempo demonstra a inexistência de teorias específicas voltadas para esta disciplina e reafirma a necessidade dos estudiosos utilizarem teorias de outros campos para embasar as pesquisas nesta área, conforme já haviam constatado Bird et al. (2002).

\subsection{Estratégias de investigação}

Das estratégias de investigação possíveis em estudos qualitativos, identificou-se as seguintes modalidades nos artigos analisados: (i) estudo teórico, (ii) estudo de caso e (iii) estudo narrativo. Apresenta-se, na sequência, a conceituação e análise de cada uma das estratégias de investigação utilizadas, buscando compreender de que forma estas foram empregadas nos estudos qualitativos sobre gestão estratégica em empresas familiares. 


\subsubsection{Estudo teórico}

Identificou-se que 13 artigos (o correspondente a 59,09\% do total) são estudos teóricos, os quais buscaram, por meio de revisões qualitativas de literatura, propor modelos ou frameworks teóricos. Chama a atenção a quantidade de estudos com este objetivo, mas considera-se que talvez esta escolha seja justificada pelo fato de representarem uma oportunidade de atualização para o pesquisador quanto a tudo que está sendo estudado sobre o tema de pesquisa, relacionando este à evolução teórica do campo do estudo.

\subsubsection{Estudo de caso}

O estudo de caso, segundo Yin (2010), é uma forma de se fazer pesquisa social empírica quando se investiga um fenômeno atual inserido no contexto de vida-real, sendo que as fronteiras existentes entre estes não são bem perceptíveis. Ele possibilita que o pesquisador aprofunde o conhecimento de situações não definidas suficientemente, sendo muito produtivo para instigar o entendimento e propor hipóteses e questões para a pesquisa, podendo ter como objeto de estudo desde uma organização até um processo específico (MATTAR, 1996).

Yin (2010) vai além, defendendo que um estudo de caso busca aprofundar-se na compreensão de determinado evento, processo ou projeto, através da quantidade de variáveis existentes pela riqueza do fenômeno e a dimensão do contexto da vida real. Os estudos de caso são generalizações a proposições teóricas, e não a populações ou universos; com isto o estudo de caso não representa uma amostragem, mas sim a expansão de teorias proporcionando um aprofundamento do conhecimento teórico em determinada área, que é o objeto de estudo (YIN, 2010).

Dentre os artigos analisados, observou-se a realização de estudos de caso em 5 pesquisas. Cabe destacar o artigo de Aguilar e Lema (2011) onde, utilizando como estratégia de investigação estudos de casos múltiplos, os autores estudaram 4 empresas hoteleiras de portes pequeno e médio (entre 11 e 100 empregados respectivamente), com mais de 5 anos de operação e situadas no México. Tendo em vista que a pesquisa tinha como objetivo analisar a cultura empresarial e a influência desta na gestão estratégica em empresas familiares, para estruturar os estudos de casos, capturando adequadamente os contextos implícitos às empresas pesquisadas, os pesquisadores optaram pela adoção de técnicas de coleta de dados combinadas, a saber: entrevistas estruturadas e em profundidade, análise de documentos e observações in loco.

\subsubsection{Estudo narrativo}

O estudo narrativo, de acordo com Labov (1972), caracteriza-se por uma narrativa como método de recapitulação da experiência passada, combinando uma sequência verbal de cláusulas para uma sequência de evento que infere-se 
que realmente ocorreram. Nos últimos anos, este tipo de investigação tem sido aplicado também nas Ciências Sociais em geral, especialmente no domínio da investigação sobre empreendedorismo, como os estudos de O'Connor (2002) e Pitt (1998) (JUN et al., 2013). Johansson (2004) complementa que o surgimento do uso explícito das narrações nas Ciências Sociais decorre do fato de que contar histórias é, muitas vezes, a forma mais natural de descrever a experiência passada.

Nos artigos analisados, o estudo de Jun et al. (2013) utilizou as histórias para investigar a interação dos empresários na área rural da China em relação ao seu ambiente institucional. Para tanto, os autores realizaram uma análise das reportagens publicadas e transmitidas por um dos mais populares meios de comunicação da China, a China Central Television (CCTV) em um programa especial que contou histórias sobre empreendedores rurais. Assim, os autores assistiram às histórias transmitidas pelo programa e analisaram as transcrições que foram fornecidas pela CCTV para levantar os dados necessários para a condução da pesquisa.

\subsection{Técnicas de coleta de dados}

Conforme Dresch, Lacerda e Antunes (2015), a escolha das técnicas de coleta de dados a serem adotadas em uma pesquisa varia em decorrência dos objetivos e do método definidos para a mesma. Dentre as técnicas de coleta de dados existentes, algumas são mais utilizadas nas pesquisas relacionadas aos processos de gestão, a saber: documental, bibliográfica, entrevistas, grupos focais, questionários e observações.

A Tabela 2 apresenta as técnicas de coleta de dados utilizadas nos 9 artigos empíricos analisados. É possível observar que as 4 diferentes técnicas empregadas foram utilizadas de maneira combinada (20 utilizações no total) nas pesquisas realizadas. As entrevistas foram utilizadas em 8 estudos, seguido pela pesquisa documental, em 7 estudos, as observações, em 4 estudos e, por fim, os grupos focais em 1 estudo. Vale ressaltar, ainda, que o artigo que apresenta uma abordagem metodológica mista teve a análise restrita apenas à etapa qualitativa do estudo (desconsiderando a aplicação da survey), visto ser este o foco da presente pesquisa. 
Tabela 2 - Técnicas de coleta de dados

\begin{tabular}{l|c|r|r}
\hline \multicolumn{1}{c|}{ Técnica } & $\mathbf{N}^{\mathbf{0}}$ Artigos & \multicolumn{1}{c|}{$\%$} & \multicolumn{1}{c}{$\%$ acumul. } \\
\hline Entrevistas & 8 & $40,00 \%$ & $40,00 \%$ \\
\hline Pesquisa Documental & 7 & $35,00 \%$ & $75,00 \%$ \\
\hline Observação & 4 & $20,00 \%$ & $95,00 \%$ \\
\hline Grupos focais & 1 & $5,00 \%$ & $100,00 \%$ \\
\hline Total & $\mathbf{2 0}$ & $\mathbf{1 0 0 , 0 0 \%}$ & \\
\hline
\end{tabular}

Fonte: elaborado pelos autores.

$\mathrm{Na}$ sequência, cada técnica é conceituada e analisada, buscando compreender de que forma foram empregadas nos estudos qualitativos sobre gestão estratégica em empresas familiares analisados.

\subsubsection{Entrevistas}

A entrevista é uma técnica na qual ocorre uma interação entre o investigador e o investigado, por meio de um diálogo assimétrico, onde o primeiro busca a coleta de dados e o segundo apresenta-se como fonte de informação, ao responder às perguntas formuladas por aquele pesquisador (GIL, 1999). Esta técnica é adequada quando pretende-se captar a percepção, o conhecimento e a expectativa dos entrevistados acerca de determinado assunto.

As entrevistas qualitativas, de acordo com Sampieri, Collado e Lucio (2006), são mais flexíveis e abertas, sendo caracterizadas por uma conversa entre uma pessoa e outra ou outras. Lakatos e Marconi (2010) complementam, afirmando que a entrevista é um encontro entre duas pessoas, a fim de que uma delas obtenha informações acerca de determinado assunto, mediante uma conversa de natureza profissional. A entrevista não é uma simples conversa, mas sim uma conversação orientada a um objetivo definido: coletar, por meio do interrogatório, os dados para realização de uma pesquisa (CERVO; BERVIAN, 2002).

Analisando os artigos deste estudo, que utilizaram a entrevista como técnica de coleta de dados, observa-se que todos eles tiveram como objetivo obter um relato em profundidade, compreendendo a fundo o fenômeno investigado. A entrevista semiestruurada (combinação de questões "fechadas" e "abertas"), foi a modalidade preferida pelos pesquisadores, uma vez que permite investigar temas de maneira mais ampla e aprofundada, indo além do que é possível por meio da utilização somente das tradicionais questões "fechadas". 


\subsubsection{Pesquisa documental}

A pesquisa documental utiliza qualquer tipo de documentação que possa fornecer dados para um estudo, sendo alguns exemplos registros oficiais, dados estatísticos, relatórios e materiais audiovisuais (CASARIN; CASARIN, 2012). Este tipo de pesquisa consiste em utilizar-se de dados que ainda não foram tratados analiticamente, como relatórios e informações da empresa (GIL, 1990). Conforme Lakatos e Marconi (2009), na pesquisa documental a fonte de coleta de dados é restrita a documentos, podendo estes serem escritos ou não. Barros e Lehfeld (2010) consideram que a análise documental constituise de uma técnica valiosa de abordagem de dados qualitativos e apresenta vantagens, como suas fontes estáveis de informação e servem de base para diferentes estudos.

Entre os estudos analisados, o artigo de Jones et al. (2013) utilizou a pesquisa documental para coleta de dados públicos, do arquivo da empresa investigada e um arquivo solicitado ao Museu Marítimo de Liverpool. Os autores analisaram sistematicamente os relatórios financeiros anuais relativos a Bibby (empresa analisada no estudo) e suas controladas, referente ao período entre 1971 e 2010. A análise forneceu dados robustos sobre mudanças nas capacidades de longo prazo da Bibby. Os dados externos foram importantes para compreender como a empresa se desenvolveu ao longo dos seus mais de 200 anos de existência, bem como para verificar a veracidade das informações obtidas na pesquisa.

\subsubsection{Observações}

Para fins da coleta de dados de uma pesquisa, observar um fenômeno é muito mais do que somente acessá-lo de maneira visual, é a combinação integrada de sensações obtidas por meio dos sentidos, das percepções e significados decorrentes da interação destes com o ambiente da pesquisa (GRAY, 2012).

A observação direta, envolve um olhar sistemático sobre a dinâmica do fenômeno pesquisado, bem como o registro, a análise e a interpretação das peculiaridades. A observação permite ao pesquisador ir além de opiniões ou interpretações particulares, capturando determinadas características e peculiaridades de um fenômeno, que muitas vezes não são claramente percebidas pelos respectivos atores deste (DRESCH; LACERDA; ANTUNES, 2015).

Conforme Gray (2012), existem 4 abordagens de observação possíveis: (i) observação aberta; (ii) observação oculta; (iii) observação participante e (iv) observação não participante, determinadas pelo nível de proximidade e de interação que o pesquisador estabelece com o campo da pesquisa. A validade científica da técnica de coleta de dados, está diretamente relacionada com ao 
rigor metodológico com que a observação é planejada e executada durante a pesquisa.

Cabe salientar que nos artigos analisados, todas as pesquisas que utilizaram a observação direta como técnica de coleta de dados, o fizeram como uma técnica complementar às entrevistas ou aos grupos focais. Dentre os artigos analisados, o estudo de Kotlar e De Massiss (2013) é um exemplo claro do uso complementar de técnicas de coleta de dados, onde os pesquisadores realizaram 76 entrevistas com membros de 19 empresas integrantes da amostra, bem como usaram a observação direta das reuniões das famílias e dos negócios quando da definição das metas das empresas.

\subsubsection{Grupos focais}

O grupo focal é uma técnica de coleta de dados que consiste na realização de entrevistas em grupo, onde um moderador é responsável pela condução e o seu objetivo é a discussão de um tópico específico (VERGARA, 2005). A utilização deste tipo de técnica permite ao pesquisador analisar a interação dentro do grupo, onde os participantes influenciam uns aos outros pelas respostas, às ideias e colocações no decorrer da discussão, estimuladas por comentários e/ou questões fornecidas pelo moderador (o pesquisador ou outra pessoa). Os dados fundamentais produzidos pela técnica são transcritos das discussões do grupo, acrescidos de anotações e reflexões do moderador e outros observadores, caso existam (OLIVEIRA; FREITAS, 2010).

O grupo focal é recomendado para orientar e referenciar a investigação em novos campos; gerar hipóteses com base na percepção dos informantes; avaliar diferentes situações de pesquisa ou populações de estudo; desenvolver planos de entrevistas ou questionários; possibilitar interpretações dos resultados dos participantes a partir de estudos iniciais e gerar informações adicionais a um estudo de larga escala (OLIVEIRA; FREITAS, 2010).

Na amostra selecionada, apenas o artigo de O'Regan et al. (2010) utilizou a técnica de grupo focal para coleta de dados. Por meio de uma reunião com um grupo de consultores de empresas familiares, os pesquisadores objetivaram obter uma visão geral sobre a gestão estratégica e o processo de tomada de decisão em empresas familiares. Vale ressaltar, ainda, que esta foi apenas uma das formas de coleta de dados adotadas pelos autores, que a combinaram com a aplicação de entrevistas em profundidade com proprietários e fundadores de empresas familiares, bem como pesquisadores com expertise na temática.

Cabe ressaltar que na maioria dos artigos analisados verificou-se a utilização combinada de pelo menos duas destas técnicas de coleta de dados (entrevistas, pesquisa documental, observações e grupos focais), o que é certamente uma vantagem do método qualitativo, pois permite ao pesquisador acessar diferentes ângulos ou perspectivas da questão pesquisada por meio da utilização de múltiplas fontes de evidência. 


\subsection{Principais limitações metodológicas apresentadas nos estudos}

Por meio da análise das considerações finais dos artigos selecionados para este estudo, observou-se que 10 deles $(45,45 \%)$ não citaram limitações referentes ao método qualitativo utilizado, sendo que destes, 8 classificam-se como estudos teóricos.

Os demais artigos mencionam diversas limitações com relação ao método qualitativo de investigação, explicitadas a seguir com as respectivas frequências de ocorrências: tamanho/restrição da amostra (6); dificuldade para generalização dos resultados (5); restrita captura da heterogeneidade das empresas pesquisadas (3); distância entre a investigação ou pesquisa e a prática de gestão (3); necessidade de testagem e refinamento (estatístico e empírico) das conclusões das pesquisas ou modelos propostos (2); restrições na coleta de dados em empresas familiares (tempo, acesso, disponibilidade, documentação, etc.) (2); falta ou concentração em um único método ou escala para medição (2); especificidade dos contextos sócio culturais pesquisados (1); ética do pesquisador com relação aos sujeitos (1); abordagens de pesquisa utilizadas pouco criativas e inovadoras (1); necessidade de aprofundar o estudo (1); resultados obtidos pouco abrangentes (1); rigor metodológico (1); alcance da região pesquisada (1); falta de uma rede forte entre pesquisadores e instituições que estudam empresas familiares (1); dificuldade na investigação dos efeitos combinados do ambiente interno e dos fatores externos das empresas (1).

Embora as limitações apresentadas não sejam inéditas aos pesquisadores, quando analisadas de forma mais ampla e integrada, remetem à necessidade de conferir maior confiabilidade, validade e generalizabilidade aos métodos qualitativos (CRESSWELL, 2010). A confiabilidade da pesquisa, pressupõe que diferentes pesquisadores, quando em situações contextuais parecidas, consigam produzir semelhantes entendimentos e formulações sobre o que está sendo estudado. Para garantir, portanto, que o foco da análise concentre-se no fenômeno pesquisado propriamente dito, conferindo estabilidade às conclusões e, portanto, maior confiabilidade ao estudo, utiliza-se técnicas de triangulação das informações (múltiplas fontes e múltiplas ferramentas de coleta de dados), treinamento dos pesquisadores como entrevistadores, se for o caso, ou mesmo a adoção de planos ou padrões de intervenção no campo, previamente definidos (GRAY, 2012).

Medidas para garantir a validade das pesquisas são tradicionalmente usadas nos métodos quantitativos e implicam na utilização de alguns procedimentos pelo pesquisador para verificar a precisão dos resultados e das conclusões obtidas (CRESSWLL, 2010). No que se refere à abordagem qualitativa, pelo menos 2 modalidades de validação mostram-se oportunas: validade interna e validade externa. A validade interna refere-se à reflexão crítica do pesquisador sobre as análises realizadas e a sua influência sobre a interpretação da realidade pesquisada. Além disso, os resultados da pesquisa podem ser validados por meio de medidas como o retorno dos dados para 
a verificação dos pesquisados (precisão e fidelidade das interpretações do pesquisador), o envolvimento de outros pesquisadores para auditar o protocolo metodológico adotado ou mesmo fracionando a análise dos dados em etapas, para checar a consistência intrínseca (GRAY, 2012). A validade externa está diretamente relacionada a questão da generalização e implica na replicação dos resultados obtidos na pesquisa em diferentes contextos, amostragens e populações alvo, acreditando nas similaridades destes aspectos.

Considerando-se que a riqueza e o valor da pesquisa qualitativa residem exatamente no acesso e no entendimento profundo de contexto ou fenômenos particulares, o que permite ao pesquisador a construção de hipóteses a respeito, para Lincoln e Guba (1994, p. 124) "se existe uma 'verdadeira' generalização, é a de que não pode haver generalização". Neste sentido, vale ressaltar que a escolha do método a ser adotado (qualitativo, quantitativo ou misto) deve privilegiar a análise dos aspectos contextuais envolvidos e do propósito da pesquisa (GRAY, 2012).

Em contraponto ao mencionado anteriormente, no que se refere à generalizabilidade da pesquisa qualitativa, é possível pensar que estudos de casos múltiplos permitem algumas generalizações teóricas mais amplas (YIN, 2010). Mesmo assim, este entendimento não é unânime entre os estudiosos e certamente exige do pesquisador um esforço adicional no sentido de documentar os procedimentos metodológicos qualitativos adotados na pesquisa.

Mesmo considerando a importância de discutir-se as limitações apontadas nos artigos analisados, no sentido de conferir maior plausibilidade ao método qualitativo e para o avanço do conhecimento, cabe destacar que as limitações identificadas são intrínsecas ao método e, portanto, menos relacionadas especificamente às pesquisas realizadas e que foram base para os artigos analisados.

\section{CONSIDERAÇÕES FINAIS}

Este artigo buscou responder à seguinte questão: como são desenvolvidas as pesquisas qualitativas sobre gestão estratégica em empresas familiares? Para responder a esta pergunta, foram estabelecidos 4 objetivos, os quais foram alcançados por meio da realização deste estudo.

O primeiro objetivo consistiu em verificar o que foi publicado sobre gestão estratégica em empresas familiares no período entre 2010 e 2015. Para alcançar este objetivo, realizou-se a busca de artigos por palavras-chave nas bases de dados EBSCO Host e ISI Web of Knowledge, onde foram selecionados 39 artigos que versaram sobre essa temática. Na sequência, os artigos foram analisados, a fim de identificar aqueles que adotaram uma abordagem metodológica qualitativa, dando origem a uma amostra de 22 artigos, sendo 21 qualitativos e 1 misto. A partir da análise desses artigos, emergiram 5 principais 
blocos temáticos, a saber: gestão estratégica, governança corporativa, gestão operacional, gestão de pessoas e cultura organizacional e gestão financeira.

No que se refere às abordagens teóricas utilizadas, verificou-se que $40,90 \%$ dos artigos não utilizaram teoria para embasamento dos estudos, enquanto que os outros artigos utilizaram 12 abordagens teóricas diferentes. A teoria dominante nos estudos é a Visão Baseada em Recursos (utilizada em 21,05\% dos estudos), seguida pelas Capacidades Dinâmicas, Teoria do Comportamento da Firma, Teoria Institucional e Teoria Prospectiva (10,53\% dos estudos cada) e a Teoria da Imagem, Teoria da Aprendizagem Organizacional, Teoria da Contingência, Teoria da Escolha Estratégica, Teoria da Regulamentação, Teoria da Troca Social e Teoria dos Stakeholders (utilizadas em $5,26 \%$ dos estudos cada).

O terceiro objetivo buscou analisar as estratégias de pesquisa e as técnicas de coleta de dados utilizadas no desenvolvimento das pesquisas qualitativas. No que se refere às estratégias de investigação, destacaram-se os estudos teóricos (13 artigos), estudos de caso (5 artigos) e o estudo narrativo (1 artigo); no que tange às técnicas de coleta de dados, observou-se a condução de entrevistas (8 artigos), pesquisa documental (7 artigos), observações (4 artigos) e grupos focais (1 artigo). Nesse sentido, os estudos teóricos apresentamse como principal estratégia de investigação utilizada nos artigos analisados $(59,09 \%)$, destacando-se que a coleta de dados, em geral, é realizada por meio da combinação de pelo menos duas das técnicas mencionadas, sendo a entrevista a técnica de coleta de dados mais utilizada.

Finalizando o estudo, o quarto objetivo consistiu em identificar as limitações metodológicas apontadas pelos autores nos artigos investigados. Entre as principais limitações, destacam-se o tamanho/restrição da amostra, a dificuldade ou impossibilidade de generalização dos resultados, a restrita captura da heterogeneidade das empresas pesquisadas, a distância entre a investigação ou pesquisa e a prática de gestão, a necessidade de testagem e refinamento (estatístico e empírico) das conclusões das pesquisas ou modelos propostos, restrições na coleta de dados em empresas familiares (tempo, acesso, disponibilidade, documentação) entre outras. Vale ressaltar que essas limitações são mais intrínsecas ao método qualitativo do que específicas das pesquisas analisadas, o que é reforçado inclusive, por diversos pesquisadores especialistas em metodologia de pesquisa.

Embora os objetivos estabelecidos para este estudo tenham sido alcançados, ele apresenta algumas limitações que devem ser destacadas. A primeira refere-se ao fato do estudo ter focado apenas em publicações em inglês ou espanhol. Seria interessante, para avanço da pesquisa, a realização desta mesma análise em revistas e conferências brasileiras, buscando identificar as pesquisas qualitativas que vêm sendo realizadas sobre gestão estratégica em empresas familiares em nível nacional. Outra limitação diz respeito à análise exclusiva de artigos publicados em periódicos, desprezando as publicações em 
eventos científicos. Assim, sugere-se que pesquisas futuras realizem a análise de artigos disponíveis em outros outlets, além de periódicos.

É importante ressaltar, ainda, que este estudo é resultado da seleção de artigos por meio de palavras-chave específicas em busca realizada no dia 23 de outubro de 2015. Como a ciência está em constante evolução, novos estudos podem ter sido publicados após esta data e não estão contemplados nesta análise. Dessa forma, pesquisas futuras, ainda que utilizando os mesmos procedimentos metodológicos apresentados neste estudo, possivelmente encontrarão resultados distintos dos aqui apresentados.

\section{REFERÊNCIAS}

AGUILAR, J. L. E.; LEMA, D. G. P. de. La cultura de las empresas familiares turísticas mexicanas y su influencia en la gestión. Cuadernos de Administración, v. 24, n. 42, p. 295-313, 2011.

ALTINDAG, E; ZEHIR, C.; ACAR, A.Z. Learning, entrepreneurship and innovation orientations in Turkish family-Owned Firms. Emerging Markets Journal, v.1,p. 36-47, 2011.

BARNEY, J.B; HESTERLY, W.S. Administração Estratégica e Vantagem Competitiva: casos brasileiros. São Paulo: Pearson, 2007.

BARROS, A. J. da S.; LEHFELD, N. A. de S. Fundamentos de metodologia científica. 3. ed. São Paulo: Pearson, 2010.

BERNS, G.S.; LAIBSON, D.; LOEWENSTEIN, G. Intertemporal choice-Toward an integrative framework. Trends in Cognitive Sciences, v. 11, n.11, p. 482-488, 2007.

BERVIAN, P. A.; CERVO, A.L. Metodologia Científica. São Paulo: Pretence Hall, 2002.

BIRD, B. et al.. Family business research: The evolution of an academic field. Family

Business Review, v. 15, n. 4, p. 337-350, 2002.

BOYD, B.; HOLLENSEN, S. Strategic management of a family-owned airline : Analysing the absorptive capacity of Cimber Sterling Group A/S. Journal of Family Business Strategy, v. 3, n. 2, p. 70-78, 2012.

BRUTON, G. D.; AHLSTRON, D.; LI, H.L. Institutional Theory and Entrepreneurship: Where Are We Now and Where Do We Need to Move in the Future?

Entrepreneurship Theory and Practice. v. 34, p. 421-440, 2010.

CALDAS, M. P.; TONELLI, M. J.; LACOMBE, B. M. B. Espelho, Espelho Meu: Metaestudo da Produção Científica em Recursos Humanos nos ENANPADs da Década de 90. In: EnANPAD, 2002, Anais... ANPAD, 2002. 
CASARIN, H. de C. S.; CASARIN, S. J. Pesquisa científica: da teoria à prática.

Curitiba: InterSaberes, 2012.

CASTRO, C. de M. A prática da pesquisa. 2. ed. São Paulo: Pearson Prentice Hall, 2006.

CHRISMAN, J. J. et al. Family involvement, family influence, and family centered non economic goals in small firms. Entrepreneurship Theory and Practice, v. 36, n. 2, p. 267-293, 2012.

CHRISMAN, J. J. et al. The Influence of Family Goals, Governance, and Resources on Firm Outcomes. Entrepreunership: Theory and Practice, p. 1249-1262, 2013.

CHRISMAN, J. J.; CHUA, J.H.; SHARMA, P. Trends and directions in the development of a strategic management theory of the family firm. Entrepreneurship Theory and Practice, v. 29, n. 5, p. 555-575, 2005.

CRESWELL, J.W. Projeto de Pesquisa: Métodos Qualitativo, Quantitativo e Misto. 3. ed. São Paulo: Bookman, 2010.

DASPIT, J. J.; LONG, R. G. Mitigating Moral Hazard in Entrepreneurial Networks: Examining Structural and Relational Social Capital in East Africa. Entrepreunership: Theory and Practice, p. 1343-1350, 2014.

DE CLERCQ, D.; BELAUSTEGUIGOITIA, I. Intergenerational strategy involvement and family firms' innovation pursuits: The critical roles of conflict management and social capital. Journal of Family Business Strategy, 2015.

DE MASSIS, A. et al. Family Business Studies: An annotated bibliography. Edward Elgar Publishing Inc, 2012.

DETIENNE, D. R.; CHIRICO, F. Exit Strategies in Family Firms: How Socioemotional wealth drives the threshold of Performance. Entrepreunership: Theory and Practice, p. 1297-1319, 2013.

DiMAGGIO, P. J.; POWELL, W. W. The Iron Cage Revisited: Institutional Isomorphism and Collective Rationality in Organizational Fields. American Sociological Review. v. 48, p. 147-160, 1983.

DRESCH, A.; LACERDA, D.P.; ANTUNES, J.A.V. Design Science Research: Método de pesquisa para avanço da ciência e tecnologia. São Paulo, Bookman, 2015.

EISENHARDT, K.; MARTIN, J. A. Dynamic Capabilities: What Are They? Strategic Management Journal, v. 21, p. 1105-1121, 2000.

GIL, A. C. Métodos e técnicas de pesquisa social. 5. ed. São Paulo: Atlas, 1999.

GIL, A. C. Técnicas de pesquisa em economia. 2. ed. São Paulo: Atlas, 1990. 
GÓMEZ-MEJÍA, L. R.; NUÑEZ-NIQUEL, M.; GUTIERREZ, I. The Role of Family Ties in Agency Contracts. Academy of Management Journal. v. 44, p. 81-95, 2001.

GRAY, D. E. Pesquisa no Mundo Real. 2. ed. Porto Alegre: Penso, 2012.

HOLT, D. T. Strategic decisions within family firms: understanding the controlling family's receptivity to internationalization. Entrepreunership:Theory \& Practice, p. 1145-1152, 2012.

JASKIEWICZ, P.; LUCHAK, A. A. Explaining Performance Differences Between Family Firms With Family and Nonfamily CEOs: It's the Nature of the Tie to the Family That Counts! Entrepreunership: Theory and Practice, p. 1361-1368, 2013.

JOHANSSON, A. W. Narrating the Entrepreneur, International Small Business Journal, v. 22, p. 273-293, 2004.

JONES, O. et al. Dynamic capabilities in a sixth-generation family firm: Entrepreneurship and the Bibby Line. Business History, p. 37-41, 2013.

JUN, Y. et al. Rural Entrepreneurship in an Emerging Economy: Reading Institutional Perspectives from Entrepreneur Stories. Journal of Small Business Management, v. 51, n. 2, p. 183-195, 2013.

KAHNEMAN, D; TVERSKY, A. Prospect theory: An analysis of decision under risk. Econometrica: Journal of the Econometric Society, n. 47, v. 2, 263-291, 1979.

LABOV, W. Transformation of Experience in Narrative Syntax. Philadelphia: University of Pennsylvania Press, 1972.

LAKATOS, E. M.; MARCONI, M. de A. Técnicas de pesquisa: planejamento e execução de pesquisas, amostragens e técnicas de pesquisas, elaboração, análise e interpretação de dados. 7. ed. São Paulo: Atlas, 2009.

LINCOLN, Y.S; GUBA, E.G. Naturalistic Inquiry. 2. ed. Newbury Park, CA: Sage, 1994.

LIONZO, A.; ROSSIGNOLI, F. Knowledge integration in family SMEs : an extension of the 4I model. Journal of Management \& Governance, p. 583-608, 2013.

LOZANO, M. B. Strategic decisions of family firms on cash accumulation. RAE Revista de Administração de Empresas, v. 55, n. 4, p. 461-467, 2015.

LUMPKIN, G. T.; BRIGHAM, K. H. Long-Term Orientation and Intertemporal Choice in Family Firms. Entrepreunership: Theory and Practice, p. 1149-1170, 2011.

LUMPKIN, G. T.; STEIER, L.; WRIGHT, M. Strategic entrepreneurship in family business. Strategic Entrepreneurship Journal, v. 5, p. 285-306, 2011. 
MALHOTRA, N.K. Introdução à pesquisa de marketing. São Paulo: Prentice Hall,2005.

MALHOTRA, N. K. Pesquisa de marketing: uma orientação aplicada. 6.ed. Porto Alegre: Bookman, 2012.

MATTAR, F. N. Pesquisa de marketing. São Paulo: Atlas, 1996.

MAYS, N. Qualitative Research: rigour and qualitative research. British Medical Journal, n. 311, p. 109-22, 1995.

MEMILI, E.; WELSH, D. H. B.; LUTHANS, F. Going beyond research on goal setting: a proposed role for organizational psychological capital of family firms. Entrepreunership: Theory and Practice, p. 1289-1297, 2013.

MEYER, J. W.; ROWAN, B. Institutionalized Organizations: Formal Structure as Myth and Ceremony, in The New Institutionalism in Organizational Analysis. Eds. W. W. Powell and P. J. DiMaggio. Chicago: University of Chicago Press, p. 41-62, 1991.

NAG, R.; HAMBRICK, D. C.; CHEN, M. J. What is strategic management, really? Inductive derivation of a consensus definition of the field. Strategic Management Journal, v. 28, n. 9, p. 935-955, 2007.

O'REGAN, N. O. et al. Strategic Thinking in Family Businesses. Strategic Change, v. 19 , p. 57-76, 2010.

OLIVEIRA, M.; FREITAS, H. Focus group: instrumentalizando o seu planejamento. In. GODOI, C. K.; BANDEIRA-DE-MELLO, R.; SILVA, A. B. da (Org.) Pesquisa Qualitativa em Estudos Organizacionais. Paradigmas, Estratégias e Métodos. São Paulo: Saraiva, 2010.

ROESCH, S. M. A. Projetos de estágio e de pesquisa em administração: guia para estágios, trabalhos de conclusão, dissertações e estudos de caso. 3. ed. São Paulo: Atlas, 2009

ROMERO, A. D. M. et al.Strategic orientations and their relationship with performance:a case of a Mexican family firm.Academy of Strategic Management Journal, v.13, p. 1-21, 2014.

SAMPIERI, R. H.; COLLADO, C. F.; LUCIO, P. B. Metodologia de Pesquisa. 3. ed. São Paulo: McGraw-Hill, 2006.

SHARMA, P.; CHRISMAN, J. J.; CHUA, J. H. Strategic management of the family business: Past research and future challenges. Family Business Review, 10, 1-36, 1997.

SHARMA, P.; CHRISMAN, J. J.; GERSICK, K.5 years of family business review:an outlook on the past and perspectives for the future. Family Business Review,v. 25, p. 5-15,2012. 
SHARMA, P.; CHUA, J. H. Asian family enterprises and family business research. Asia Pacific Journal of Management, v. 30, p. 641-656, 2013.

SHARMA, P.; SALVATO, C. Commentary: exploiting and exploring new opportunities over Life Cycle Stages of Family. Entrepreunership: Theory and Practice, p. 1199-1206, 2011.

SOUZA, S. A. de; REINERT, J. N.; SPROSSER, R. L. Pesquisa brasileira em Administração: um meta-estudo em temáticas do período 2000-2009. In: II Encontro de Ensino e Pesquisa em Administração e Contabilidade, 2009, Anais... ANPAD, 2009.

STEWARD, A.; MINER, A.S. The Prospects for Family Business in Research universities. Journal of Family Business Strategy, v. 2, p. 3- 14, 2011.

VERGARA, S. C. Métodos de pesquisa em administração. São Paulo: Atlas, 2005.

WRIGHT, M. et al. Family enterprise and context. Entrepreunership: Theory and Practice, p. 1247-1261, 2014.

YIN, R. K. Estudo de caso: planejamento e métodos. 4. ed. Porto Alegre: Bookman, 2010.

YU, J. et al. Reading Institutional Perspectives from Entrepreneur Stories. Journal of Small Business Management, v. 51, n. 2, p. 183-195, 2013. 